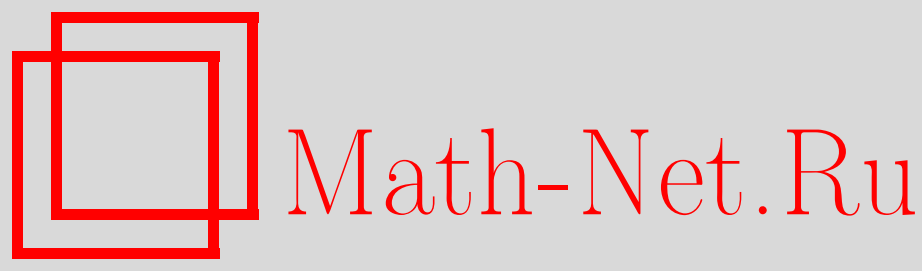

Цзе-Сюн Мо, Обобщенный принцип неопределенности и энтропия черной дыры Шварцшильда-анти-де Ситтера с глобальным монополем, обусловленным спиновыми полями, ТМФ, 2012, том 171, номер 1, 154-161

DOI: https://doi.org/10.4213/tmf6891

Использование Общероссийского математического портала Math-Net.Ru подразумевает, что вы прочитали и согласны с пользовательским соглашением http://www . mathnet.ru/rus/agreement

Параметры загрузки:

IP : 54.198 .187 .58

26 апреля 2023 г., 16:50:08

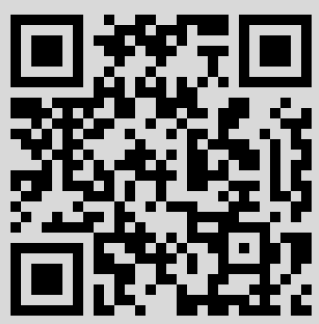




\title{
ОБОБЩЕННЫЙ ПРИНЦИП НЕОПРЕДЕЛЕННОСТИ И ЭНТРОПИЯ ЧЕРНОЙ ДЫРЫ ШВАРЦШИЛЬДА-АНТИ-ДЕ СИТТЕРА С ГЛОБАЛЬНЫМ МОНОПОЛЕМ, ОБУСЛОВЛЕННЫМ СПИНОВЫМИ ПОЛЯМИ
}

\begin{abstract}
Квантовая энтропия черной дыры Шварцшильда-анти-де Ситтера с глобальным монополем, обусловленным полями произвольного спина $s \leqslant 2$, исследована с использованием скорректированной плотности состояний согласно обобщенному принципу неопределенности. Показано, что квантовая энтропия зависит не только от характеристик черной дыры, но и от спина поля и множителя, связанного с гравитационной поправкой. Существование энергетического масштаба нарушения симметрии уменьшает полную энтропию. Кроме того, показано, что вклад гравитационных взаимодействий в энтропию является очень важным и им нельзя пренебрегать.
\end{abstract}

Ключевые слова: обобщенный принцип неопределенности, энтропия, спиновые поля, черная дыра.

\section{1. ВВЕДЕНИЕ}

Изучение термодинамики черных дыр стало одной из интереснейших проблем теоретической физики с тех пор, как Бекенстайн и Хокинг [1], [2] исследовали энтропию черной дыры, сравнивая физику черной дыры с термодинамикой. Согласно Бекенстайну и Хокингу энтропия четырехмерной черной дыры пропорциональна площади поверхности горизонта. В 1985 году 'т Хофт [3] развил знаменитую модель кирпичной стены, которая широко использовалась для вычисления энтропии черных дыр различных типов. Однако, чтобы контролировать расходимость на горизонте, в модели кирпичной стены искусственным путем было введено обрезание. Если обрезание удовлетворяет некоторому условию, то энтропия сводится к результату Бекенстайна-Хокинга. В работах [4]-[7] с помощью метода евклидова интеграла по траекториям было обнаружено, что кроме результата 'т Хофта

*Institute of Theoretical Physics, Zhanjiang Normal University, Zhanjiang, China. E-mail: mojiexiong@gmail.com 
энтропия все же содержит логарифмически расходящийся член. Кроме того, было обнаружено, что логарифмические поправки к энтропии Бекенстайна-Хокинга, обусловленные спиновыми полями, распространяющимися на фоне черной дыры, зависят от спина [8]-[16]. Было показано, что обобщенный принцип неопределенности, т. е. $\Delta X \Delta P \geqslant \hbar\left[1+\lambda(\Delta P)^{2}\right] / 2$, вводит новое, ранее не известное определение плотности состояний [17]-[27]. Согласно обобщенному принципу неопределенности плотность состояний принимает вид $d^{3} X d^{3} Y /(2 \pi \hbar)^{3}\left(1+\lambda P^{2}\right)^{3}$, которое сильно отличается от выражения $d^{3} X d^{3} Y /(2 \pi \hbar)^{3}$, отвечающего принципу неопределенности Гейзенберга. Здесь $\lambda$ - множитель, обусловленный гравитационной поправкой, а $P$ - импульс частицы. С использованием скорректированной плотности состояний была вычислена энтропия для различных черных дыр [28]-[36].

В настоящей работе мы сконцентрируем свое внимание на черной дыре Шварцшильда-анти-де Ситтера с глобальным монополем. Глобальный монополь, введенный Бариолой и Виленкиным [37], является одним из наиболее важных топологических дефектов, обусловленных космологическими фазовыми переходами, когда симметрии в ранней Вселенной спонтанно нарушались. Как утверждали Бариола и Виленкин, монополи могут порождать эффекты, доступные наблюдению, а также могут влиять на образование галактик [37]. Большая энергия в голдстоуновском поле, окружающем глобальный монополь, предполагает, что он может порождать сильное гравитационное поле, а это чрезвычайно интересно и означает, что обобщенный принцип неопределенности может играть важную роль. Используя различные виды метрики, авторы работ [38] и [39] независимо исследовали некоторые свойства глобального монополя в пространстве-времени анти-де Ситтера. В работе [40] была вычислена энтропия статической черной дыры Шварцшильда-анти-де Ситтера с глобальным монополем в рамках модели кирпичной стены и было обнаружено, что квантовая энтропия содержит как квадратично расходящийся член, так и зависящий от спина логарифмически расходящийся член. В работе [41] обсуждается энтропия, обусловленная скалярной черной дырой с глобальным монополем во всех порядках по планковской длине. Однако пока никто из исследователей не применял обобщенный принцип неопределенности для изучения энтропии черной дыры Шварцшильда-анти-де Ситтера с глобальным монополем, обусловленным спиновыми полями. В настоящей работе мы применяем обобщенный принцип неопределенности для вычисления квантовой энтропии черной дыры Шварцшильдаанти-де Ситтера с глобальным монополем, обусловленным полями произвольного спина $s \leqslant 2$.

\section{2. ПРОСТРАНСТВО-ВРЕМЯ И ПОЛЕВЫЕ УРАВНЕНИЯ}

Элемент длины статической черной дыры Шварцшильда-анти-де Ситтера с глобальным монополем имеет вид [39]

$$
d s^{2}=B d t^{2}-B^{-1} d r^{2}-r^{2} d \theta^{2}-r^{2} \sin ^{2} \theta d \varphi^{2},
$$

где $B=1-\eta^{2}-2 M / r-\Lambda r^{2} / 3, M$ - масса черной дыры, $\Lambda<0$ - космологическая постоянная, $\eta$ - энергетический масштаб нарушения симметрии. Горизонт событий можно найти из уравнения $B=0$, которое имеет три вещественных корня при $\sqrt[3]{9 M^{2} \Lambda}<1-\eta^{2}<0$. Кроме того, три вещественных корня задаются следующим 
образом [40]:

$$
\begin{gathered}
r_{\mathrm{H}}=\sqrt{\frac{2\left(1-\eta^{2}\right)^{3}}{|\Lambda|^{3}}} \cos \frac{\alpha}{3}, \quad r_{1}=\sqrt{\frac{2\left(1-\eta^{2}\right)^{3}}{|\Lambda|^{3}}} \cos \left(\frac{\alpha}{3}+\frac{\pi}{3}\right), \\
r_{-1}=\sqrt{\frac{2\left(1-\eta^{2}\right)^{3}}{|\Lambda|^{3}}} \cos \left(\frac{\alpha}{3}-\frac{\pi}{3}\right)
\end{gathered}
$$

где $\cos \alpha=-3 M \sqrt{\left(1-\eta^{2}\right)^{3} /|\Lambda|^{3}}, r_{\mathrm{H}}$ соответствует внешнему горизонту, а $r_{1}-$ внутреннему. Используя три корня уравнения $B=0$, величину $B$ можно переписать как

$$
B=-\frac{\Lambda}{3 r}\left(r-r_{\mathrm{H}}\right)\left(r-r_{1}\right)\left(r-r_{-1}\right)
$$

Площадь поверхности гравитационного горизонта черной дыры имеет вид

$$
\kappa=-\frac{\Lambda}{6 r_{\mathrm{H}}}\left(r_{\mathrm{H}}-r_{1}\right)\left(r_{\mathrm{H}}-r_{-1}\right)
$$

Нулевые векторы тетрады для пространства-времени (1) выбираются как

$$
l^{\mu}=\left[B^{-1}, 1,0,0\right], \quad n^{\mu}=\frac{1}{2}[1,-B, 0,0], \quad m^{\mu}=\frac{1}{\sqrt{2} r}\left[0,0,1, \frac{i}{\sin \theta}\right] .
$$

Используя формулу Ньюмана-Пенроуза [42], из соотношений (1) и (5) получаем ненулевые спиновые коэффициенты и компоненты вейлевского тензора:

$$
\alpha=-\beta=-\frac{\operatorname{ctg} \theta}{2 \sqrt{2} r}, \quad \rho=-\frac{1}{r}, \quad \mu=-\frac{B}{2 r}, \quad \gamma=\frac{B^{\prime}}{4}, \quad \psi_{2}=-\frac{M}{r^{3}}-\frac{\eta^{2}}{6 r^{2}},
$$

откуда видно, что метрика (1) является метрикой Петрова типа D. B этом типе пространства-времени уравнения для поля вейлевского нейтрино $(s=1 / 2)$, электромагнитного поля $(s=1)$, безмассового поля Рариты-Швингера $(s=3 / 2)$ и гравитационного поля $(s=2)$ можно скомбинировать следующим образом [43]:

$$
\begin{aligned}
& \{[D-2 s \rho-\bar{\rho}](\Delta-2 s \gamma+\mu)-[\delta-\bar{\alpha}-(2 s-1) \beta] \times \\
& \left.\quad \times(\bar{\delta}-2 s \alpha)-(2 s-1)(s-1) \psi_{2}\right\} \Phi_{+s}=0 \\
& \{[\Delta+(2 s-1) \gamma-\bar{\gamma}+2 s \mu+\bar{\mu}](D-\rho)- \\
& \left.\quad-[\bar{\delta}+\bar{\beta}+(2 s-1) \alpha](\delta+2 s \beta)-(2 s-1)(s-1) \psi_{2}\right\} \phi_{-s}=0
\end{aligned}
$$

где $D=l^{\mu} \partial \mu, \Delta=n^{\mu} \partial \mu, \delta=m^{\mu} \partial \mu$ - производные по направлениям. Уравнение (7) является уравнением для спиновых состояний с $p=s$, а уравнение (8) - 
для спиновых состояний с $p=-s$. Подставляя (5) и (6) в уравнения (7) и (8) и используя ВКБ-приближение при $\Phi_{p}=\exp \left[-i E t+i S_{p}(r, \theta, \varphi)\right]$, можно переписать уравнения (7) и (8) как

$$
B^{-1} E^{2}-B P_{r}^{2}-\frac{1}{r^{2}} P_{\theta}^{2}-\frac{1}{r^{2} \sin ^{2} \theta}\left(P_{\varphi}+p \cos \theta\right)^{2}+\xi(r, p)=0,
$$

где $P_{\mu}=\partial_{\mu} S_{p}-$ сопряженные импульсы, а

$$
\begin{aligned}
\xi(r, p)=\frac{p}{r^{2}} & -\frac{\eta^{2}}{3 r^{2}}(p+1)(2 p+1)-\frac{2 \Lambda}{3}(p+1)(2 p+1)+ \\
& +\frac{s-p}{r^{4}}\left[r^{2}-2 s M r-\eta^{2} r^{2}+\frac{\Lambda r^{4}}{3}(2 s-3)\right]
\end{aligned}
$$

Из уравнения (9) можно получить

$$
P^{2}=-\left[\frac{E^{2}}{B}+\xi(r, p)\right] .
$$

Вблизи горизонта событий импульс стремится к бесконечности, поэтому целесообразно использование предела геометрической оптики, а использование ВКБ-приближения оправданно, справедливость чего была показана в работе [3].

\section{3. ВЫЧИСЛЕНИЕ ЭНТРОПИИ}

В данном разделе мы воспользуемся скорректированной плотностью состояний для вычисления квантовой энтропии черной дыры Шварцшильда-анти-де Ситтера с глобальным монополем, обусловленным полями произвольного спина $s \leqslant 2$. Согласно обобщенному принципу неопределенности число квантовых состояний с энергией, не превосходящей $E$, имеет вид

$$
\begin{aligned}
g(E) & =\frac{1}{(2 \pi)^{3}} \sum_{p} \int \frac{d r d \theta d \varphi d P_{r} d P_{\theta} d P_{\varphi}}{\left(1+\lambda P^{2}\right)^{3}}= \\
& =\frac{2}{3 \pi} \sum_{p} \int B^{1 / 2}\left[\frac{E^{2}}{B}+\xi(r, p)\right]^{3 / 2}\left[1-\lambda\left(\frac{E^{2}}{B}+\xi(r, p)\right)\right]^{-3} r^{2} d r,
\end{aligned}
$$

где представлены интегралы по импульсному пространству и интегралы по $\theta$ и $\varphi$. Теперь из формулы (12) получаем, что число квантовых состояний с энергией, не превосходящей $E$, является конечным вблизи горизонта. Таким образом, с помощью числа квантовых состояний можно получить сходящуюся энтропию. Это демонстрирует огромное преимущество обобщенного принципа неопределенности по сравнению с моделью кирпичной стены.

Свободная энергия системы имеет вид

$$
-\beta F= \pm \sum_{q} \ln \left[1 \pm e^{-\beta E_{q}}\right],
$$

где $\beta$ - обратная температура, $q$ - набор квантовых чисел, знак плюс соответствует ферми-случаю, а знак минус - бозе-случаю. Пренебрегая малыми членами, из 
скорректированной плотности состояний (12) можно получить свободную энергию:

$$
F=-\int_{0}^{\infty} \frac{g(E) d E}{e^{\beta E} \mp 1}=-\frac{2}{3 \pi} \sum_{p} \int \frac{r^{2} d r}{\sqrt{B}} \int_{0}^{\infty}\left(\frac{E^{3}}{B^{3 / 2}}+\frac{3 E \xi(r, p)}{2 \sqrt{B}}+\frac{3 \lambda E^{5}}{B^{5 / 2}}\right) \frac{d E}{e^{\beta E} \mp 1} .
$$

Перейдем к следующим этапам вычисления энтропии. Сначала возьмем интеграл по $E$ в (13), что дает для бозонов и фермионов соответственно

$$
\begin{aligned}
& F_{\mathrm{B}}=-\int \frac{r^{2} d r}{\sqrt{B}}\left[\frac{2 \omega \pi^{3}}{45 \beta^{4} B^{3 / 2}}+\frac{\pi \sum_{p} \xi(r, p)}{6 \beta^{2} B^{1 / 2}}+\frac{16 \lambda \omega \pi^{5}}{63 \beta^{6} B^{5 / 2}}\right], \\
& F_{\mathrm{F}}=-\int \frac{r^{2} d r}{\sqrt{B}}\left[\frac{7 \omega \pi^{3}}{180 \beta^{4} B^{3 / 2}}+\frac{\pi \sum_{p} \xi(r, p)}{12 \beta^{2} B^{1 / 2}}+\frac{31 \lambda \omega \pi^{5}}{126 \beta^{6} B^{5 / 2}}\right],
\end{aligned}
$$

где $\omega=\sum_{p} 1$ - вырождение, обусловленное спином. Для скалярного поля $\omega=1$, а для поля вейлевского нейтрино, электромагнитного поля, безмассового поля Рариты-Швингера и гравитационного поля $\omega=2$. Теперь, рассматривая сферическую оболочку от $r_{\mathrm{H}}+\varepsilon$ до $r_{\mathrm{H}}+L$ как область интегрирования в координатном пространстве и выписав интеграл по $r$ в соотношении (13), мы получаем окончательно выражение для свободной энергии для бозонов и фермионов соответственно

$$
\begin{aligned}
& F_{\mathrm{B}}=-\frac{2 \omega \pi^{3}}{45 \beta^{4}}\left[\frac{9 r_{\mathrm{H}}^{4}}{\Lambda^{2}\left(r_{\mathrm{H}}-r_{1}\right)^{2}\left(r_{\mathrm{H}}-r_{-1}\right)^{2} \varepsilon}+\frac{18}{\Lambda^{2}} \frac{r_{\mathrm{H}}^{3}\left(-r_{\mathrm{H}} r_{1}-r_{\mathrm{H}} r_{-1}+2 r_{1} r_{-1}\right)}{\left(r_{\mathrm{H}}-r_{1}\right)^{3}\left(r_{\mathrm{H}}-r_{-1}\right)^{3}} \ln \frac{L}{\varepsilon}\right]- \\
& -\frac{\pi}{6 \beta^{2}} \frac{2 \eta^{2} r_{\mathrm{H}}(s+1)(2 s+1)+2 \Lambda r_{\mathrm{H}}^{3}\left(2 s^{2}+3 s+2\right)+12 s^{2} M-6 s r_{\mathrm{H}}}{\Lambda\left(r_{\mathrm{H}}-r_{1}\right)\left(r_{\mathrm{H}}-r_{-1}\right)} \ln \frac{L}{\varepsilon}- \\
& -\frac{16 \omega \pi^{5} \lambda}{63 \beta^{6}}\left[-\frac{27 r_{\mathrm{H}}^{5}}{2 \Lambda^{3}\left(r_{\mathrm{H}}-r_{1}\right)^{3}\left(r_{\mathrm{H}}-r_{-1}\right)^{3} \varepsilon^{2}}+\frac{27 r_{\mathrm{H}}^{4}\left(r_{\mathrm{H}}^{2}+2 r_{\mathrm{H}} r_{1}+2 r_{\mathrm{H}} r_{-1}-5 r_{1} r_{-1}\right)}{\Lambda^{3}\left(r_{\mathrm{H}}-r_{1}\right)^{4}\left(r_{\mathrm{H}}-r_{-1}\right)^{4} \varepsilon}-\right. \\
& -\left(\frac{27 r_{\mathrm{H}}^{3}\left(2 r_{\mathrm{H}}^{4}+8 r_{1} r_{\mathrm{H}}^{3}+8 r_{-1} r_{\mathrm{H}}^{3}+2 r_{1}^{2} r_{\mathrm{H}}^{2}+2 r_{-1}^{2} r_{\mathrm{H}}^{2}-22 r_{1} r_{-1} r_{\mathrm{H}}^{2}\right.}{\Lambda^{3}\left(r_{\mathrm{H}}-r_{1}\right)^{5}\left(r_{\mathrm{H}}-r_{-1}\right)^{5}}-\right. \\
& \left.\left.-\frac{\left.10 r_{1} r_{-1}^{2} r_{\mathrm{H}}-10 r_{1}^{2} r_{-1} r_{\mathrm{H}}+20 r_{1}^{2} r_{-1}^{2}\right)}{\Lambda^{3}\left(r_{\mathrm{H}}-r_{1}\right)^{5}\left(r_{\mathrm{H}}-r_{-1}\right)^{5}}\right) \ln \frac{L}{\varepsilon}\right] \text {, } \\
& F_{\mathrm{F}}=-\frac{7 \omega \pi^{3}}{180 \beta^{4}}\left[\frac{9 r_{\mathrm{H}}^{4}}{\Lambda^{2}\left(r_{\mathrm{H}}-r_{1}\right)^{2}\left(r_{\mathrm{H}}-r_{-1}\right)^{2} \varepsilon}+\frac{18}{\Lambda^{2}} \frac{r_{\mathrm{H}}^{3}\left(-r_{\mathrm{H}} r_{1}-r_{\mathrm{H}} r_{-1}+2 r_{1} r_{-1}\right)}{\left(r_{\mathrm{H}}-r_{1}\right)^{3}\left(r_{\mathrm{H}}-r_{-1}\right)^{3}} \ln \frac{L}{\varepsilon}\right]- \\
& -\frac{\pi}{12 \beta^{2}} \frac{2 \eta^{2} r_{\mathrm{H}}(s+1)(2 s+1)+2 \Lambda r_{\mathrm{H}}^{3}\left(2 s^{2}+3 s+2\right)+12 s^{2} M-6 s r_{\mathrm{H}}}{\Lambda\left(r_{\mathrm{H}}-r_{1}\right)\left(r_{\mathrm{H}}-r_{-1}\right)} \ln \frac{L}{\varepsilon}- \\
& -\frac{31 \omega \pi^{5} \lambda}{126 \beta^{6}}\left[-\frac{27 r_{\mathrm{H}}^{5}}{2 \Lambda^{3}\left(r_{\mathrm{H}}-r_{1}\right)^{3}\left(r_{\mathrm{H}}-r_{-1}\right)^{3} \varepsilon^{2}}+\frac{27 r_{\mathrm{H}}^{4}\left(r_{\mathrm{H}}^{2}+2 r_{\mathrm{H}} r_{1}+2 r_{\mathrm{H}} r_{-1}-5 r_{1} r_{-1}\right)}{\Lambda^{3}\left(r_{\mathrm{H}}-r_{1}\right)^{4}\left(r_{\mathrm{H}}-r_{-1}\right)^{4} \varepsilon}-\right. \\
& -\left(\frac{27 r_{\mathrm{H}}^{3}\left(2 r_{\mathrm{H}}^{4}+8 r_{1} r_{\mathrm{H}}^{3}+8 r_{-1} r_{\mathrm{H}}^{3}+2 r_{1}^{2} r_{\mathrm{H}}^{2}+2 r_{-1}^{2} r_{\mathrm{H}}^{2}-22 r_{1} r_{-1} r_{\mathrm{H}}^{2}\right.}{\Lambda^{3}\left(r_{\mathrm{H}}-r_{1}\right)^{5}\left(r_{\mathrm{H}}-r_{-1}\right)^{5}}-\right. \\
& \left.\left.-\frac{\left.-10 r_{1} r_{-1}^{2} r_{\mathrm{H}}-10 r_{1}^{2} r_{-1} r_{\mathrm{H}}+20 r_{1}^{2} r_{-1}^{2}\right)}{\Lambda^{3}\left(r_{\mathrm{H}}-r_{1}\right)^{5}\left(r_{\mathrm{H}}-r_{-1}\right)^{5}}\right) \ln \frac{L}{\varepsilon}\right] \text {. }
\end{aligned}
$$


Наконец, вычислим полную энтропию $S$ с помощью формулы $S=\beta^{2} \partial F / \partial \beta$. Пренебрегая малыми членами, получим для бозонов и фермионов соответственно

$$
\begin{aligned}
S_{\mathrm{B}}= & -\frac{\omega A_{\mathrm{H}}}{360 \beta_{\mathrm{H} \varepsilon}}+\omega\left[\frac{2 \pi r_{\mathrm{H}}}{45 \beta_{\mathrm{H}}}+\frac{\Lambda r_{\mathrm{H}}^{2}}{540}\right] \ln \frac{L}{\varepsilon}+\frac{3 s(1-s)-\eta^{2}\left(-s^{2}+3 s+1\right)-\Lambda r_{\mathrm{H}}^{2}\left(s^{2}+3 s+2\right)}{18} \ln \frac{L}{\varepsilon}+ \\
& +\frac{\omega \lambda}{336}\left\{\frac{A_{\mathrm{H}} \pi}{\beta_{\mathrm{H}}^{2} \varepsilon^{2}}+\frac{\pi \Lambda}{3 \beta_{\mathrm{H}} \varepsilon}\left[15 \frac{\left(1-\eta^{2}\right)^{3}}{\Lambda^{3}}-4 r_{\mathrm{H}}^{2}\right]+\frac{1}{6}\left[\frac{6\left(1-\eta^{2}\right)^{3}}{\Lambda}+4 r_{\mathrm{H}}^{2} \Lambda^{2}-\frac{20\left(1-\eta^{2}\right)^{6}}{\Lambda^{4} r_{\mathrm{H}}^{2}}\right] \ln \frac{L}{\varepsilon}\right\}, \\
S_{\mathrm{F}}= & -\frac{7 \omega A_{\mathrm{H}}}{2880 \beta_{\mathrm{H}} \varepsilon}+\frac{7 \omega}{8}\left[\frac{2 \pi r_{\mathrm{H}}}{45 \beta_{\mathrm{H}}}+\frac{\Lambda r_{\mathrm{H}}^{2}}{540}\right] \ln \frac{L}{\varepsilon}+\frac{3 s(1-s)-\eta^{2}\left(-s^{2}+3 s+1\right)-\Lambda r_{\mathrm{H}}^{2}\left(s^{2}+3 s+2\right)}{36} \ln \frac{L}{\varepsilon}+ \\
& +\frac{31 \omega \lambda}{10752}\left\{\frac{A_{\mathrm{H}} \pi}{\beta_{\mathrm{H}}^{2} \varepsilon^{2}}+\frac{\pi \Lambda}{3 \beta_{\mathrm{H}} \varepsilon}\left[15 \frac{\left(1-\eta^{2}\right)^{3}}{\Lambda^{3}}-4 r_{\mathrm{H}}^{2}\right]+\frac{1}{6}\left[\frac{6\left(1-\eta^{2}\right)^{3}}{\Lambda}+4 r_{\mathrm{H}}^{2} \Lambda^{2}-\frac{20\left(1-\eta^{2}\right)^{6}}{\Lambda^{4} r_{\mathrm{H}}^{2}}\right] \ln \frac{L}{\varepsilon}\right\},
\end{aligned}
$$

где $A_{\mathrm{H}}=4 \pi r_{\mathrm{H}}^{2}-$ площадь поверхности горизонта. В данных соотношениях мы выбрали обратную температуру $\beta$ так, чтобы она соответствовала температуре Хокин-

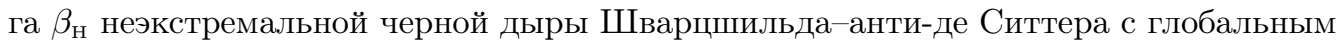
монополем. Третий член зависит от спина поля, а четвертый член, который обусловлен поправкой к обобщенному принципу неопределенности, отражает эффекты гравитационных взаимодействий.

В модели кирпичной стены чистое расстояние от кирпичной стены до горизонта равно $l_{p}=\int_{r}^{r+\varepsilon} \sqrt{-g_{r r}} d r$, в то время как параметры ультрафиолетового и инфракрасного обрезания можно положить равными $l_{p}^{2}=2 \epsilon^{2} / 15$ и $\Gamma^{2}=L \epsilon^{2} / \varepsilon$ соответственно. Однако полученная на основании обобщенного принципа неопределенности самая малая длина $\Delta X$ равна $\sqrt{\lambda}$. Поэтому можно взять $\sqrt{\lambda}$ в качестве чистого расстояния от сферической оболочки до горизонта, т. е. $\sqrt{\lambda}=\int_{r}^{r+\varepsilon} \sqrt{-g_{r r}} d r$. Тогда с учетом обобщенного принципа неопределенности соотношения для $S_{\mathrm{B}}$ и $S_{\mathrm{F}}$ можно переписать следующим образом:

$$
\begin{aligned}
S_{\mathrm{B}}= & -\frac{\omega A_{\mathrm{H}}}{360 \lambda \pi}+\omega\left(\frac{2 \pi r_{\mathrm{H}}}{45 \beta_{\mathrm{H}}}+\frac{\Lambda r_{\mathrm{H}}^{2}}{540}\right) \ln \frac{2 \Gamma^{2}}{15 \lambda}+ \\
& +\frac{3 s(1-s)-\eta^{2}\left(-s^{2}+3 s+1\right)-\Lambda r_{\mathrm{H}}^{2}\left(s^{2}+3 s+2\right)}{18} \ln \frac{2 \Gamma^{2}}{15 \lambda}+\frac{\omega A_{\mathrm{H}}}{336 \pi \lambda}, \\
S_{\mathrm{F}}= & -\frac{7 \omega A_{\mathrm{H}}}{2880 \lambda \pi}+\frac{7 \omega}{8}\left(\frac{2 \pi r_{\mathrm{H}}}{45 \beta_{\mathrm{H}}}+\frac{\Lambda r_{\mathrm{H}}^{2}}{540}\right) \ln \frac{2 \Gamma^{2}}{15 \lambda}+ \\
& +\frac{3 s(1-s)-\eta^{2}\left(-s^{2}+3 s+1\right)-\Lambda r_{\mathrm{H}}^{2}\left(s^{2}+3 s+2\right)}{36} \ln \frac{2 \Gamma^{2}}{15 \lambda}+\frac{31 \omega A_{\mathrm{H}}}{10752 \pi \lambda} .
\end{aligned}
$$

Отсюда видно, что значения энтропии для бозе- и ферми-случаев похожи, за исключением того, что различные поля подчиняются различным статистическим законам. Хотя четвертый член во многом похож на первый, они имеют различные следствия. Отношение четвертого члена к первому равно 15/14 для бозе-случая и 465/392 для ферми-случая, откуда следует, что четвертый член важен и им не следует пренебрегать.

\section{4. ЗАКЛЮЧЕНИЕ И ОБСУЖДЕНИЕ}

При исследовании квантовой энтропии черной дыры Шварцшильда-анти-де Ситтера с глобальным монополем, обусловленным полем вейлевского нейтрино, электромагнитным полем, безмассовым полем Рариты-Швингера и гравитационным полем, мы учли скорректированную плотность состояний, обусловленную обобщенным 
принципом неопределенности. Квантовая энтропия зависит не только от характеристик черной дыры, но также от спина поля и гравитационного поправочного множителя. Поправка, обусловленная обобщенным принципом неопределенности, имеет важное значение для энтропии. Заметим, что существование энергетического масштаба нарушения симметрии уменьшает полную энтропию.

Можно записать энтропию (15) черной дыры Шварцшильда-анти-де Ситтера с глобальным монополем, обусловленным скалярным полем вейлевского нейтрино, электромагнитным полем, безмассовым полем Рарита-Швингера и гравитационным полем, более явно как

$$
S= \begin{cases}\frac{A_{\mathrm{H}}}{360 \lambda \pi}+\left(\frac{2 \pi r_{\mathrm{H}}}{45 \beta_{\mathrm{H}}}+\frac{\Lambda r_{\mathrm{H}}^{2}}{540}\right) \ln \frac{2 \Gamma^{2}}{15 \lambda}-\frac{\eta^{2}+2 \Lambda r_{\mathrm{H}}^{2}}{18} \ln \frac{2 \Gamma^{2}}{15 \lambda}+\frac{A_{\mathrm{H}}}{336 \pi \lambda}, & s=0, \\ \frac{14 A_{\mathrm{H}}}{2880 \lambda \pi}+\frac{7}{4}\left(\frac{2 \pi r_{\mathrm{H}}}{45 \beta_{\mathrm{H}}}+\frac{\Lambda r_{\mathrm{H}}^{2}}{540}\right) \ln \frac{2 \Gamma^{2}}{15 \lambda}-\frac{9 \eta^{2}+15 \Lambda r_{\mathrm{H}}^{2}-3}{144} \ln \frac{2 \Gamma^{2}}{15 \lambda}+\frac{62 A_{\mathrm{H}}}{10752 \pi \lambda}, & s=\frac{1}{2}, \\ \frac{2 A_{\mathrm{H}}}{360 \lambda \pi}+2\left(\frac{2 \pi r_{\mathrm{H}}}{45 \beta_{\mathrm{H}}}+\frac{\Lambda r_{\mathrm{H}}^{2}}{540}\right) \ln \frac{2 \Gamma^{2}}{15 \lambda}-\frac{3 \eta^{2}+6 \Lambda r_{\mathrm{H}}^{2}}{18} \ln \frac{2 \Gamma^{2}}{15 \lambda}+\frac{2 A_{\mathrm{H}}}{336 \pi \lambda}, & s=1, \\ \frac{14 A_{\mathrm{H}}}{2880 \lambda \pi}+\frac{7}{4}\left(\frac{2 \pi r_{\mathrm{H}}}{45 \beta_{\mathrm{H}}}+\frac{\Lambda r_{\mathrm{H}}^{2}}{540}\right) \ln \frac{2 \Gamma^{2}}{15 \lambda}-\frac{9+13 \eta^{2}+35 \Lambda r_{\mathrm{H}}^{2}}{144} \ln \frac{2 \Gamma^{2}}{15 \lambda}+\frac{62 A_{\mathrm{H}}}{10752 \pi \lambda}, & s=\frac{3}{2}, \\ \frac{2 A_{\mathrm{H}}}{360 \lambda \pi}+2\left(\frac{2 \pi r_{\mathrm{H}}}{45 \beta_{\mathrm{H}}}+\frac{\Lambda r_{\mathrm{H}}^{2}}{540}\right) \ln \frac{2 \Gamma^{2}}{15 \lambda}-\frac{6+3 \eta^{2}+12 \Lambda r_{\mathrm{H}}^{2}}{18} \ln \frac{2 \Gamma^{2}}{15 \lambda}+\frac{2 A_{\mathrm{H}}}{336 \pi \lambda}, & s=2, \\ \frac{29 A_{\mathrm{H}}}{5040 \lambda \pi}+\left(\frac{2 \pi r_{\mathrm{H}}}{45 \beta_{\mathrm{H}}}-\frac{59 \Lambda r_{\mathrm{H}}^{2}}{540}-\frac{\eta^{2}}{18}\right) \ln \frac{2 \Gamma^{2}}{15 \lambda}, & s=\frac{1}{2}, \\ \frac{857 A_{\mathrm{H}}}{80640 \lambda \pi}+\left(\frac{7 \pi r_{\mathrm{H}}}{90 \beta_{\mathrm{H}}}-\frac{109 \Lambda r_{\mathrm{H}}^{2}}{1080}-\frac{\eta^{2}}{16}+\frac{1}{48}\right) \ln \frac{2 \Gamma^{2}}{15 \lambda}, & s=\frac{1}{6}, \\ \frac{29 A_{\mathrm{H}}}{2520 \lambda \pi}+\left(\frac{4 \pi r_{\mathrm{H}}}{45 \beta_{\mathrm{H}}}-\frac{89 \Lambda r_{\mathrm{H}}^{2}}{270}-\frac{\eta^{2}}{6}\right) \ln \frac{2 \Gamma^{2}}{15 \lambda}, & s=1, \\ \frac{857 A_{\mathrm{H}}}{80640 \lambda \pi}+\left(\frac{7 \pi r_{\mathrm{H}}}{90 \beta_{\mathrm{H}}}-\frac{259 \Lambda r_{\mathrm{H}}^{2}}{1080}-\frac{13 \eta^{2}}{144}-\frac{1}{16}\right) \ln \frac{2 \Gamma^{2}}{15 \lambda}, & s=\frac{3}{2}, \\ \frac{29 A_{\mathrm{H}}}{2520 \lambda \pi}+\left(\frac{4 \pi r_{\mathrm{H}}}{45 \beta_{\mathrm{H}}}-\frac{179 \Lambda r_{\mathrm{H}}^{2}}{270}-\frac{\eta^{2}}{6}-\frac{1}{3}\right) \ln \frac{2 \Gamma^{2}}{15 \lambda}, & s=2 .\end{cases}
$$

Когда $\eta=0, \Lambda=0$, пространство-время редуцируется к пространству-времени Шварцшильда. Тогда выражения (15) можно можно записать в виде

$$
\begin{aligned}
& S_{\mathrm{B}}=-\frac{\omega A_{\mathrm{H}}}{360 \lambda \pi}+\frac{\omega}{90} \ln \frac{2 \Gamma^{2}}{15 \lambda}+\frac{s(1-s)}{6} \ln \frac{2 \Gamma^{2}}{15 \lambda}+\frac{\omega A_{\mathrm{H}}}{336 \pi \lambda}, \\
& S_{\mathrm{F}}=-\frac{7 \omega A_{\mathrm{H}}}{2880 \lambda \pi}+\frac{7 \omega}{720} \ln \frac{2 \Gamma^{2}}{15 \lambda}+\frac{s(1-s)}{12} \ln \frac{2 \Gamma^{2}}{15 \lambda}+\frac{31 \omega A_{\mathrm{H}}}{10752 \pi \lambda},
\end{aligned}
$$

которые полностью совпадают с результатом, полученным в работе [36]. Для скалярного поля первые два члена в (16) сводятся к результату работы [4], т. е.

$$
S^{\text {Solodukhin }}=\frac{A_{\mathrm{H}}}{360 \lambda \pi}+\frac{1}{90} \ln \frac{2 \Gamma^{2}}{15 \lambda}=\frac{A_{\mathrm{H}}}{48 \pi \epsilon^{2}}+\frac{1}{45} \ln \frac{\Gamma}{\epsilon} .
$$

Благодарности. Автор благодарен профессору Гу-Цян Ли за полезные обсуждения. Автор также выражает большую благодарность рецензенту за ценные замечания. 


\section{Список литературы}

[1] J. D. Bekenstein, Phys. Rev. D, 7:8 (1973), 2333-2346.

[2] S. W. Hawking, Commun. Math. Phys., 43:3 (1975), 199-220.

[3] G. 't Hooft, Nucl. Phys. B, 256:4 (1985), 727-745.

[4] S. N. Solodukhin, Phys. Rev. D, 54:6 (1996), 3900-3903, arXiv: hep-th/9601154.

[5] S. N. Solodukhin, Phys. Rev. D, 51:2 (1995), 618-621, arXiv: hep-th/9408068.

[6] S. N. Solodukhin, Phys. Rev. D, 51:2 (1995), 609-617.

[7] S. N. Solodukhin, Living Rev. Relat., 14 (2011), 8, 96 pp., arXiv: 1104.3712.

[8] Z.-H. Li, Phys. Rev. D, 62:2 (2000), 024001, 3 pp.

[9] Z.-H. Li, Modern Phys. Lett. A, 17:14 (2002), 887-897.

[10] L.-Q. Mi, Z.-H. Li, Modern Phys. Lett. A, 21:23 (2006), 1821-1827.

[11] J. Jing, M.-L. Yan, Phys. Rev. D, 64:6 (2001), 064015, 4 pp., arXiv: gr-qc/0104054.

[12] J. Jing, M. L. Yan, Phys. Rev. D, 63:8 (2001), 084028, 10 pp.

[13] Z.-H. Li, Phys. Lett. B, 643:1 (2006), 64-70.

[14] G.-Q. Li, Chin. Phys., 14:3 (2005), 468-471.

[15] G.-Q. Li, Physica A, 368:2 (2006), 425-429.

[16] G.-Q. Li, Internat. J. Modern Phys. A, 22:28 (2007), 5229-5235.

[17] A. Kempf, G. Mangano, R. B. Mann, Phys. Rev. D, 52:2 (1995), 1108-1118, arXiv: hep-th/9412167.

[18] L. N. Chang, D. M. Minic, N. Okamura, T. Takeuchi, Phys. Rev. D, 65:12 (2002), 125028, 7 pp.

[19] D. J. Gross, Phys. Rev. Lett., 60:13 (1988), 1229-1232.

[20] M. Maggiore, Phys. Lett. B, 319:1-3 (1993), 83-86, arXiv: hep-th/9309034.

[21] T. Padmanabhan, Class. Quantum Grav., 4:4 (1987), L107-L114.

[22] D. Amati, M. Ciafaloni, G. Veneziano, Phys. Lett. B, 216:1-2 (1989), 41-47.

[23] M. Maggiore, Phys. Rev. D, 49:10 (1994), 5182-5187, arXiv: hep-th/9305163.

[24] F. Scardigli, Phys. Lett. B, 452:1-2 (1999), 39-44, arXiv: hep-th/9904025.

[25] A. Kempf, G. Mangano, Phys. Rev. D, 55:12 (1997), 7909-7920, arXiv: hep-th/9612084.

[26] D. J. Gross, P. F. Mende, Nucl. Phys. B, 303:3 (1988), 407-454.

[27] E. Witten, Phys. Today, 49:4 (1996), 24-30.

[28] X. Li, Phys. Lett. B, 540:1-2 (2002), 9-13.

[29] Y.-W. Kim, Y.-J. Park, Phys. Rev. D, 77:6 (2008), 067501, arXiv: 0709.0774.

[30] M. Yoon, J. Ha, W. Kim, Phys. Rev. D, 76:4 (2007), 047501, 3 pp., arXiv: 0706.0364.

[31] W. Kim, Y.-W. Kim, Y.-J. Park, Phys. Rev. D, 74:10 (2006), 104001, 5 pp.

[32] W. Kim, Y.-W. Kim, Y.-J. Park, Phys. Rev. D, 75:12 (2007), 127501, 4 pp., arXiv: gr-qc/0702018.

[33] W.-B. Liu, Chin. Phys. Lett., 20:3 (2003), 440-443.

[34] C.-Z. Liu, Chin. Phys. Lett., 23:5 (2006), 1092-1095.

[35] F.-W. Shu, Y.-G. Shen, Chin. Phys. Lett., 24:9 (2007), 2497-2500.

[36] G.-Q. Li, EPL, 92:4 (2010), 40008.

[37] M. Barriola, A. Vilenkin, Phys. Rev. Lett., 63:4 (1989), 341-343.

[38] Y. Zhang, J.-L. Jing, Chin. Phys. Lett., 22:10 (2005), 2496-2499.

[39] Y.-W. Han, S.-Z. Yang, Chin. Phys. Lett., 22:11 (2005), 2769-2771.

[40] G.-Q. Li, J. Statist. Phys., 125:3 (2006), 753-760.

[41] F. He, F. Zhao, Chin. Phys. Lett., 26:4 (2009), 040401.

[42] E. Newman, R. Penrose, J. Math. Phys., 3:3 (1962), 566-578.

[43] S. A. Teukolsky, W. H. Press, Astrophys. J., 193 (1974), 443-461.

Поступила в редакцию 3.03.2011, после доработки 24.06.2011 